\title{
Interactions of Ficolin and Mannose-Binding Lectin with Fibrinogen/Fibrin Augment the Lectin Complement Pathway
}

\author{
Yuichi Endo $^{a}$ Naomi Nakazawa ${ }^{a}$ Daisuke Iwaki ${ }^{a}$ Minoru Takahashi ${ }^{a}$ \\ Misao Matsushita ${ }^{b}$ Teizo Fujita $^{a}$ \\ a Departments of Immunology, Fukushima Medical University School of Medicine, Fukushima and \\ ${ }^{b}$ Department of Applied Biochemistry, Tokai University, Hiratsuka, Japan
}

\section{Key Words}

Blood coagulation · Complement · Fibrin · Fibrinogen •

Ficolin $\cdot$ Lectin pathway $\cdot$ Mannose-binding lectin

\begin{abstract}
Ficolin and mannose-binding lectin (MBL) are animal lectins that are involved in innate immunity by initiating the lectin complement pathway. Here, we report that interactions between these lectins and fibrinogen/fibrin augment the lectin pathway. An ELISA revealed that recombinant mouse ficolin $A(r F c n A), r M B L-A$ and $r M B L-C$ bind to fibrinogen in a dose-dependent manner. Affinity Western blotting showed that these lectins bind to the $A \alpha-$ and $B \beta$-chains of fibrinogen and the $\alpha$-and $\beta$-chains of fibrin, but not to the $\gamma$-chain, and that rMBL-A and rMBL-C preferentially bind to the $\alpha$ - and $\beta$-chains. The $C 4$ deposition activity on Fbg-coated plates was observed by using mouse serum, and the deposition on GIcNAc-coated plates was enhanced by fibrinogen supplementation and further enhanced by the addition of thrombin. Similar effects of fibrinogen and fibrin were observed in the bindings of these lectins to a Gram-positive pathogen, Staphylococcus aureus, and in the subsequent C3 deposition on the bacteria. In particular, the lectin pathway, through MBLs, seemed to synchronize with blood coagulation. There-
\end{abstract}

fore, it is suggested that the lectin pathway collaborates with the coagulation system in the first-line host defense against pathogens under conditions such as injury and inflammation.

Copyright $\odot 2009$ S. Karger AG, Basel

\section{Introduction}

The molecular basis of innate immunity lies in the ability of firstline host defense molecules to discriminate infectious agents from self. Lectin occupies a crucial position in this system through its recognition of pathogenassociated molecular patterns exposed on pathogens. Among the animal lectins, ficolin (FCN) and mannosebinding lectin (MBL) have a unique property in activating complement via MBL-associated serine proteases (MASPs) in the lectin pathway [1-4]. Upon the bindings of FCN and MBL to pathogens, the pro-enzymes of MASPs are converted to the active forms and, in turn, MASPs activate the complement.

FCN is a group of proteins containing both a collagenlike and a fibrinogen (Fbg)-like domain [5], and the latter is responsible for a common binding specificity for $\mathrm{N}$ acetylglucosamine (GlcNAc) [6]. To date, 3 types of FCN 
have been identified in humans, L-FCN [5], M-FCN [7, 8] and $\mathrm{H}-\mathrm{FCN}$ [9], and 2 types in mice, FcnA [10] and FcnB [11]. FCN is roughly classified into 2 groups: the serum type, which includes L-FCN, H-FCN and FcnA, and the non-serum type, which includes M-FCN and FcnB [12]. We have previously demonstrated that all human FCN can form complexes with MASPs and sMAP, a truncated form of MASP-2, to consequently activate the lectin pathway $[2,13-15]$.

Recently, X-ray crystal analysis of FCN identified the 3D structure of its Fbg-like domain and the molecular architecture underlying GlcNAc-recognition, which are very similar to those of tachylectin $5 \mathrm{~A}$, a defense lectin in horseshoe crab $[6,16,17]$. Interestingly, tachylectin $5 \mathrm{~A}$, which is mainly composed of the Fbg-like domain, binds to the tetrapeptide GlyProArgPro, a well known inhibitor of blood coagulation, in addition to GlcNAc [18]. It is known that this tetrapeptide mimics the $\mathrm{N}$-terminal sequences of $\alpha$ - and $\beta$-chains of fibrin (Fbn). Tanio et al. [16] reported that the binding of M-FCN to GlcNAc was inhibited by this tetrapeptide, indicating that FCN also interacts with this tetrapeptide. Based on these observations, it seems possible that FCN can interact with Fbg and/or Fbn. In addition, our phylogenetic analysis of Fbglike sequences revealed that the Fbg-like domain of FCN is structurally related to the $\gamma$-chain of Fbg, and possibly evolved from a common ancestor $[19,20]$. This suggests the plausible ability of FCN to bind to the $\alpha$ - and $\beta$ chains, because the $N$-termini of the $\alpha$ - and $\beta$-chains interact with the ' $a$ - and b-pockets' of the $\gamma$-chain for fibrin clotting during blood coagulation.

To confirm the interaction of FCN with Fbg and/or Fbn, we assessed the binding of FcnA to Fbg- and Fbncoated microtiter plates and the $\mathrm{C} 4$ deposition activity of serum on the plates. In a comparison, the bindings of MBL-A and MBL-C to Fbg- and Fbn-coated plates were also assessed. Next, using Staphylococcus aureus as a nonself target, we assessed the effects of Fbg and Fbn on the bindings of these recognition molecules to the bacteria and on the $\mathrm{C} 3$ deposition activity of serum on the bacteria. We report here the enhanced bindings of the recognition molecules to the targets and the subsequent, augmented complement activation in the presence of Fbg and/or Fbn. We also discuss the possible collaboration of the lectin pathway with the blood coagulation system and its physiological significance in host defense of innate immunity.

\section{Materials and Methods}

Recombinant FcnA, Recombinant MBLs and Mouse Serum

The recombinant $\mathrm{F} c \mathrm{~A} \mathrm{~A}(\mathrm{rFcnA})$ was prepared as previously described [15], and used for the assays of its bindings to Fbg- and GlcNAc-BSA-coated microtiter plates and to $S$. aureus. The recombinant mouse MBLs (rMBLs), termed rMBL-A and rMBL-C, were purchased from R\&D Systems (Minneapolis, Minn., USA) and used for the similar binding assays. The pooled serum was obtained from C57BL6 mice, and used for C4- and C3-deposition assays to determine the activity of the wild type (WT) mouse serum, as described below. The pooled FcnA-deficient serum was obtained from FcnA-deficient mice, which was established by gene targeting in a C57BL6 background [manuscript in preparation]. To prepare the FcnA- and MBL-depleted serum (FcnA/ MBL-depleted serum), the FcnA-deficient serum was incubated with $1 / 10$ volume of mannan-agarose gel slurry at $4^{\circ} \mathrm{C}$ for $2 \mathrm{~h}$, and then centrifuged to remove the agarose gel.

All DNA recombination and animal studies were conducted according to the guidelines of Fukushima Medical University.

ELISA for the Bindings of Recombinant rFcnA and rMBLs to Fbg and Fbn

Microtiter plates (Sumilon, Sumitomo Bakelite, Tokyo) were coated with human Fbg ( $>80 \%$ of clottable protein; Sigma-Aldrich, St. Louis, Mo., USA) or with mouse Fbg $(>80 \%$ of clottable protein, Sigma Aldrich) at a concentration of $10 \mu \mathrm{g} / \mathrm{ml}$ in $0.25 \mathrm{M}$ Na-phosphate, $\mathrm{pH} 7.5$, and incubated overnight at $4^{\circ} \mathrm{C}$. The plates were then washed with $50 \mathrm{mM}$ Tris- $\mathrm{HCl}, \mathrm{pH} 7.5$ containing $0.15 \mathrm{M} \mathrm{NaCl}, 2 \mathrm{~mm} \mathrm{CaCl} 2$ and $0.5 \%$ Tween-20 (TBS-Ca/T) and blocked with $0.1 \% \mathrm{BSA}$ in TBS-Ca at $37^{\circ} \mathrm{C}$ for $3 \mathrm{~h}$. rFcnA, rMBL$\mathrm{A}$ and $\mathrm{rMBL}-\mathrm{C}$ were incubated in the Fbg-coated microtiter plates at amounts of 0.031 to $2 \mu \mathrm{g}$ per well in $100 \mu \mathrm{l}$ of reaction mixture, at $37^{\circ} \mathrm{C}$ for $1 \mathrm{~h}$. After washing with TBS-Ca/T, the microtiter plates were incubated with anti-FcnA [15], anti-MBL-A and antiMBL-C Abs (HyCult Biotechnology, Uden, Netherlands), followed by the biotinylated second Abs (DakoCytomation, Glostrup, Demark), and finally with the avidin-biotinylated HRP complex (ABC reagents; Vector Lab, Burlingame, Calif., USA). HRP activity was determined by incubation with TMB (KPL Co., Gaithersburg, Md., USA) and $\mathrm{H}_{2} \mathrm{O}_{2}$ at room temperature for 1-10 min, which was stopped with $1 \mathrm{M} \mathrm{H}_{3} \mathrm{PO}_{4}$. The developed color was monitored in a Multimode detector DTX880 (Beckman Coulter, Fullerton, Calif., USA) at $450 \mathrm{~nm}$. In a comparison, the bindings of above recombinants to GlcNAc and their nonspecific bindings were evaluated by using GlcNAc-BSA-coated and non-coated microtiter plates, respectively.

In a similar experiment to assess their bindings to Fbn, the Fbgcoated microtiter plates were washed with TBS-Ca/T and then treated with $20 \mathrm{mU}$ of human thrombin $(>2,000 \mathrm{NIH}$ units/mg protein; Sigma Aldrich) at $37^{\circ} \mathrm{C}$ for $30 \mathrm{~min}$ in $100 \mu \mathrm{l}$ of TBS-Ca per well. The plates were washed again, blocked and then used for the binding assay described above. In an inhibition experiment, $\mathrm{rFcnA}$, rMBL-A and rMBL-C were incubated in the Fbg-coated microtiter plates in the presence of up to $150 \mathrm{~mm}$ GlcNAc or mannose.

Affinity Western Blotting for Fbg and Fbn with rFcnA, $r M B L-A$ and $r M B L-C$ as Probes

To prepare Fbn, Fbg was treated with $20 \mathrm{mU}$ of thrombin at $37^{\circ} \mathrm{C}$ for $10 \mathrm{~min}$. The deglycosylated $\mathrm{Fbg}$ was prepared by treating 
Fbg with endoglycosidase F (endo F) as described [21]. The same amounts of Fbg, deglycosylated Fbg and Fbn were subjected to SDS-PAGE under reducing conditions, and then transferred to an Immobilon-P filter (Millipore, Billerica, Mass., USA). The filter was probed with $\mathrm{rFcnA}, \mathrm{rMBL}-\mathrm{A}$ and $\mathrm{rMBL}-\mathrm{C}$ at concentrations of $0.05 \mu \mathrm{g} / \mathrm{ml}$ in TBS-Ca/T. The bound recombinants were detected by incubation with primary Abs against FcnA, MBL-A and $\mathrm{MBL}-\mathrm{C}$. The signal was visualized by further incubation with biotinylated second Abs (DakoCytomation), ABC reagents (Vector $\mathrm{Lab}$ ), and a chemiluminescence substrate (ECL; Amersham Biosciences, Amersham, UK). The chemiluminescence image was read in a LAS-3000 (Fujifilm, Tokyo).

\section{C4 Deposition on Fbg-Coated or GlcNAc-Coated}

Microtiter Plate

To evaluate the complement activation through the lectin pathway, C4 deposition was determined by an ELISA, as previously described [15]. Briefly, up to $2 \mu \mathrm{l}$ of mouse serum was incubated in a Fbg-coated microtiter plate in $100 \mu \mathrm{l}$ of TBS-Ca at $37^{\circ} \mathrm{C}$ for $10 \mathrm{~min}$. The plate was then incubated with human $\mathrm{C} 4$ on ice for $30 \mathrm{~min}$, and the bound C4b was detected with HRPsheep anti-human $\mathrm{C} 4 \mathrm{Ab}$ (Biogenesis, Poole, UK). Color was developed using TMB (KPL Co.) and $\mathrm{H}_{2} \mathrm{O}_{2}$, and monitored in a DTX880 Multimode detector (Beckman) at $450 \mathrm{~nm}$. In another experiment to evaluate the effects of $\mathrm{Fbg} / \mathrm{Fbn}$ on $\mathrm{C} 4$ deposition on GlcNAc, $0.75 \mu \mathrm{l}$ of the mouse serum was incubated with GlcNAc-BSA-coated microtiter plates in $100 \mu \mathrm{l}$ of TBS-Ca containing various amounts of Fbg in the presence or absence of 20 $\mathrm{mU}$ thrombin at $37^{\circ} \mathrm{C}$ for $10 \mathrm{~min}$, and bound $\mathrm{C} 4 \mathrm{~b}$ was detected as above.

\section{FACS for the Bindings of $r F c n A, r M B L-A$ and $r M B L-C$ to}

S. aureus

The bindings of rFcnA, rMBL-A and rMBL-C to $S$. aureus were evaluated as previously described [14]. Briefly, $1 \times 10^{7}$ cells of heat-killed $S$. aureus were incubated with $0.45 \mu \mathrm{g}$ of $\mathrm{rFcnA}$, rMBL-A, and rMBL-C in $60 \mu \mathrm{l}$ of Hanks balanced salt solution containing $\mathrm{Ca}^{2+}$ and $\mathrm{Mg}^{2+}\left(\mathrm{HBSS}^{++}\right.$; Sigma Aldrich) at $4{ }^{\circ} \mathrm{C}$ for $1 \mathrm{~h}$. After washing with $\mathrm{HBSS}^{++}, S$. aureus was further incubated with anti-FcnA, anti-MBL-A and anti-MBL-C Abs, and then with FITC-labeled second Abs (DakoCytomation). In an inhibition experiment, rFcnA, rMBL-A and rMBL-C was incubated with 150 $\mathrm{mM}$ GlcNAc or mannose prior to incubation with the bacteria. The binding activity was evaluated on a FACScalibur flow cytometer (BD Biosciences, San Jose, Calif., USA).

In another experiment to evaluate the effects of $\mathrm{Fbg} / \mathrm{Fbn}$, the heat-killed S. aureus was pretreated with Fbg in the presence or absence of $10 \mathrm{mU}$ thrombin at $37^{\circ} \mathrm{C}$ for $20 \mathrm{~min}$ in $60 \mu \mathrm{lof} \mathrm{HBSS}^{++}$, then washed with $\mathrm{HBSS}^{++}$. S. aureus treated was then subjected to the binding assay, where it was incubated with $0.45 \mu \mathrm{g}$ of $\mathrm{rFcnA}$, rMBL-A and rMBL-C at $4{ }^{\circ} \mathrm{C}$ for $1 \mathrm{~h}$. The recombinants bound to the bacteria were detected as above.

\section{Preparation of FcnA-MASP and MBLs-MASP Complexes} from Mouse Serum

FcnA-MASP and MBLs-MASP complexes were prepared from the pooled mouse serum by GlcNAc-agarose chromatography, as the mannose- and GlcNAc-eluates, respectively. Briefly, the pooled mouse serum from WT mice was applied to a GlcNAcagarose column, and the column was extensively washed with
TBS-Ca/T. The bound fraction was eluted with $0.3 \mathrm{M}$ mannose and then with $0.3 \mathrm{M}$ GlcNAc. The 2 eluates, mannose- and GlcNAc-eluates, were dialyzed against TBS-Ca and concentrated in a centrifugal filter (Amicon Ultra-4; Millipore). The protein concentration of the eluate was determined using a BCA protein assay kit (Pierce, Rockford, Ill., USA).

\section{FACS for C3 Deposition on S. aureus}

C3 deposition was assessed as previously described [22]. Briefly, $1 \times 10^{8}$ cells of heat-killed $S$. aureus were incubated with $10 \mu \mathrm{l}$ of the pooled mouse serum in the presence or absence of $30 \mu \mathrm{g}$ Fbg and $100 \mathrm{mU}$ thrombin in $45 \mu \mathrm{l}$ of $\mathrm{HBSS}^{++}$at $37^{\circ} \mathrm{C}$ for $2 \mathrm{~min}$. The reaction was terminated by adding $1 \mathrm{ml}$ of chilled $\mathrm{HBSS}^{++}$. The $\mathrm{C} 3 \mathrm{~b}$ on the bacteria was quantified using rat anti-mouse $\mathrm{C} 3 \mathrm{~b}$ (HyCult Biotechnology) and FITC-conjugated anti-rat IgG (DakoCytomation) Abs in a FACScalibur flow cytometer (BD Biosciences). In a similar experiment to evaluate the $\mathrm{C} 3$ deposition activities of FcnA-MASPs and MBLs-MASPs complexes, FcnA/ MBL-depleted serum was supplemented with $6.0 \mu \mathrm{g}$ of the mannose and GlcNAc eluates and subjected to C3 deposition assay as above.

\section{Results}

$r F c n A$ Bound to the A $\alpha$-and B $\beta$-Chains of Fbg, and the $\alpha$ - and $\beta$-Chains of Fbn

ELISA revealed that $\mathrm{rFcnA}$ bound to the Fbg-coated microtiter plates in a dose-dependent manner (fig. 1a). A similar binding was observed when the Fbg-coated microtiter plate was treated with thrombin, in which a proportion of the coated Fbg is converted to Fbn, although the conversion was not experimentally confirmed. Another ELISA with mouse Fbg-coated plates instead of human Fbg-coated plates revealed very similar results (data not shown). As shown in figure $1 \mathrm{~b}$, affinity Western blotting demonstrated that $\mathrm{rFcnA}$ recognized the $A \alpha-$ and $\mathrm{B} \beta$-chains of human Fbg, in addition to the $\alpha$ - and $\beta$ chains of Fbn, but not the $\gamma$-chain of Fbg/Fbn. Treatment of Fbg with endo F reduced the molecular sizes of the B $\beta$ and $\gamma$-chains but did not affect the $\alpha$-chain. Treatment of Fbg with $\mathrm{O}$-glycanase (endo- $\alpha-\mathrm{N}$-acetylgalactosaminidase) in the presence of neuraminidase, which cleaves $\mathrm{O}$ linked sugars, showed no effect on the sizes of all 3 chains (data not shown). As shown in figure $1 \mathrm{~b}, \mathrm{rFcnA}$ also bound to the deglycosylated $\mathrm{B} \beta$-chain. These results indicate that $\mathrm{rFcnA}$ binds to Fbg and Fbn not through the carbohydrate moiety of $\mathrm{Fbg} / \mathrm{Fbn}$. The binding of $\mathrm{rFcnA}$ to Fbg was as high as that to GlcNAc (fig. 1c), although a precise comparison between the Fbg-coated and GlcNAccoated microtiter plates is difficult. At least, the binding ability of $\mathrm{rFcnA}$ to Fbg seems to be higher than those of rMBL-A and rMBL-C (compare fig. 1c with fig. 2a). As 

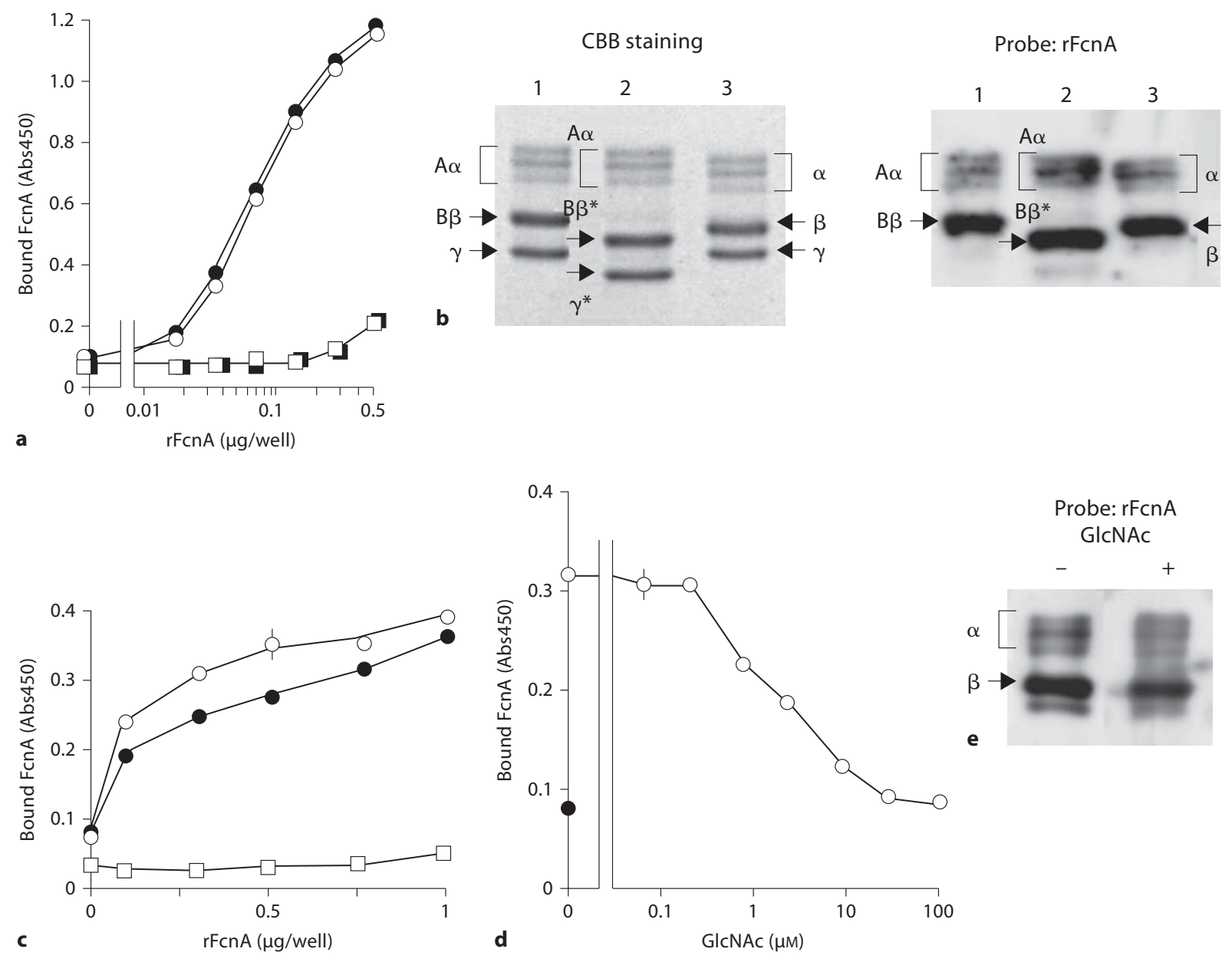

Fig. 1. Binding of $\mathrm{rFcnA}$ to $\mathrm{Fbg} / \mathrm{Fbn}$. a Dose-dependent binding of rFenA to Fbg-coated (open circle) and Fbg-coated/thrombintreated microtiter plates (closed circle), as determined by ELISA. In a control experiment, nonspecific binding of $\mathrm{rFcnA}$ to noncoated (open square) and non-coated/thrombin-treated microtiter plates (closed square) was evaluated. b Affinity Western blottings of Fbg and Fbn probed with $\mathrm{rFcnA}$. In Coomassie Brilliant Blue (CBB) staining (left panel) and affinity Western blotting (right panel), $1.2 \mu \mathrm{g}$ each of Fbg (lane 1), endo F-treated Fbg (lane 2) and Fbn (lane 3) were subjected to SDS-PAGE on $8 \%$ polyacryl-

shown in figure 1d, the binding of $\mathrm{rFcnA}$ to Fbg was inhibited by GlcNAc in a dose-dependent manner. Complete inhibition was observed at a concentration of GlcNAc exceeding $50 \mathrm{mM}$. A similar inhibitory effect of GlcNAc on the binding to Fbn was also observed in the affinity Western blotting (fig. 1e). These results confirmed the binding specificity of $\mathrm{rFcnA}$ to Fbg, and suggest that $\mathrm{rFcnA}$ recognizes Fbg and Fbn by the same or a very similar GlcNAc-binding site. amide gel under reducing conditions. The deglycosylated $\mathrm{B} \beta$ - and $\gamma$-chains are marked with asterisks. c Comparison of the binding of $\mathrm{rFcnA}$ to Fbg-coated microtiter plates (closed circle) with that to GlcNAc-BSA-coated (open circle). Open squares show results from nonspecific binding to non-coated plates. $\mathbf{d}$ Dose-dependent inhibition of GlcNAc on Fbg-binding of $r F c n A$. $r F c n A$ was used at $0.45 \mu \mathrm{g} / \mathrm{well}$. Closed circles show results from blank without $\mathrm{rFcnA}$ and GlcNAc. e Affinity Western blotting of Fbn probed with $\mathrm{rFcnA}$ in the presence of $100 \mathrm{~mm}$ GlcNAc.

\section{$r M B L-A$ and $r M B L-C$ Showed a Binding Preference} for $\alpha$-and $\beta$-Chains over $A \alpha$ - and $B \beta$-Chains

As shown in figure 2a, rMBL-A and rMBL-C bound to Fbg, although their bindings appears to be very weak as compared with those to GlcNAc. Unlike $\mathrm{rFcnA}$, the bindings of rMBLs were slightly increased by treating the Fbgcoated plates with thrombin (fig. 2b). The bindings of rMBLs to Fbg and Fbn (Fbg treated with thrombin) were reduced to $25-50 \%$ by excess amounts of GlcNAc and 


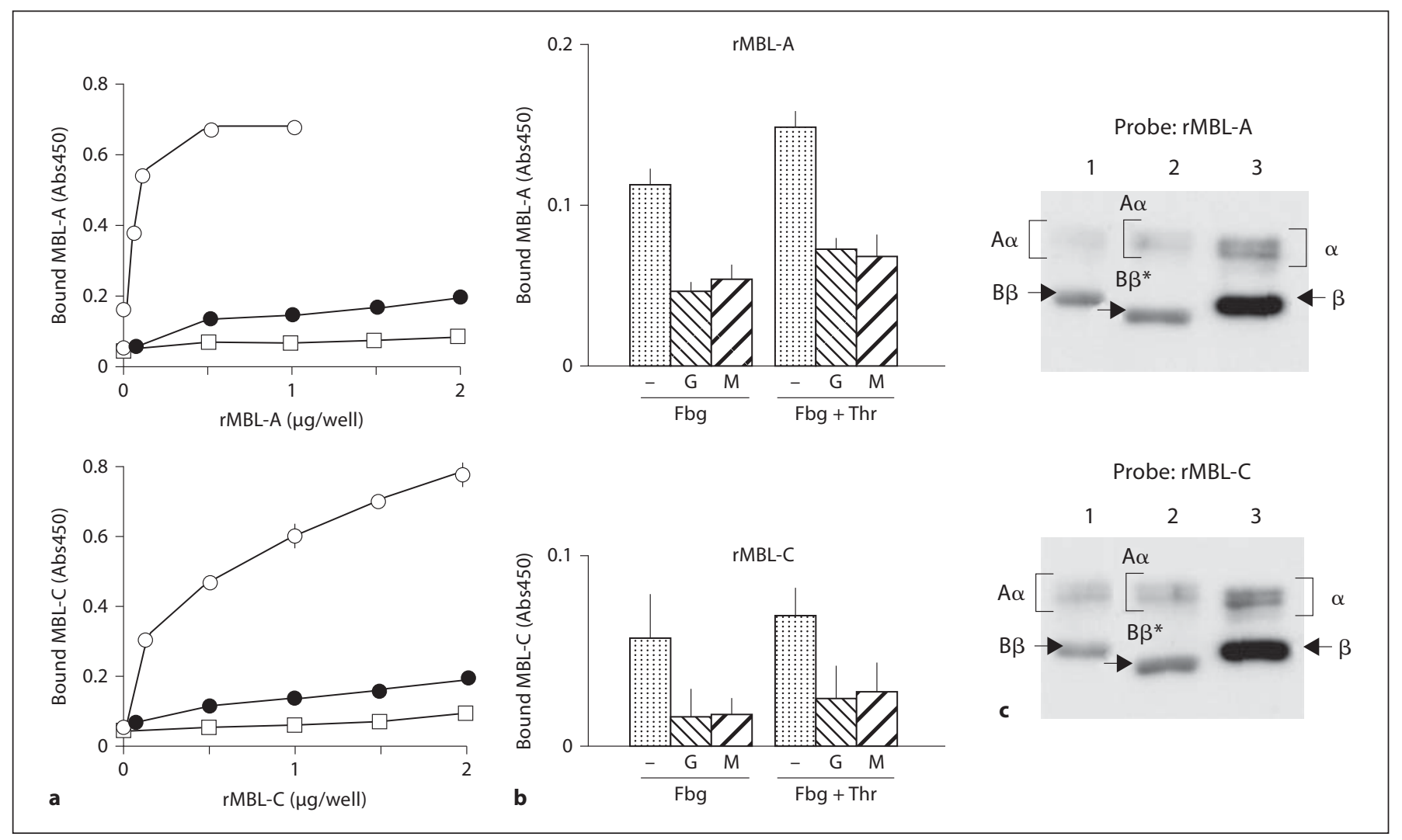

Fig. 2. Binding of rMBL-A and rMBL-C to Fbg/Fbn. a Dose-dependent binding of rMBL-A (upper panel) and rMBL-C (lower panel) to Fbg-coated (closed circle), GlcNAc-BSA-coated (open circle) and non-coated microtiter plates (open square), as determined by ELISA. b Inhibitory effects of GlcNAc $(\mathrm{G})$ and mannose $(\mathrm{M})$ on the bindings of rMBL-A and rMBL-C. $1 \mu \mathrm{g} /$ well each of rMBL-A (upper panel) and rMBL-C (lower panel) was incubated

mannose (fig. 2b), suggesting that rMBLs recognize Fbg and Fbn through similar sites to their sugar recognition. Affinity Western blotting revealed that, like $r F c n A$, rMBLs can bind to the $A \alpha-, B \beta-, \alpha$ - and $\beta$-chains, but not to the $\gamma$-chain (fig. 2c). Unlike rFcnA, rMBLs bound much more strongly to the $\alpha$ - and $\beta$-chains than to the $A \alpha$ - and $B \beta$-chains. Similar to $\mathrm{rFcnA}$, rMBL-A and rMBL-C also recognized the deglycosylated Fbg, suggesting that the recognition is not via the carbohydrate moiety of Fbg.

\section{C4 Deposition was Observed on Fbg-Coated \\ Microtiter Plates, and C4 Deposition on \\ GlcNAc-BSA-Coated Microtiter Plates was \\ Enhanced in the Presence of Fbg/Fbn}

As shown in figure $3 \mathrm{a}$, the pooled serum of WT mice revealed the $\mathrm{C} 4$ deposition activity on Fbg-coated microtiter plates, although the activity was much lower in Fbg-coated and Fbg-coated/thrombin (Thr)-treated microtiter plates in the presence of $100 \mathrm{~mm}$ of GlcNAc $(\mathrm{G})$ or mannose (M). Each binding level is expressed by subtracting the blank level without rMBLs. c Affinity Western blotting of $\mathrm{Fbg} / \mathrm{Fbn}$ probed with rMBL-A (upper panel) and rMBL-C (lower panel). The Fbg and $\mathrm{Fbn}$ were blotted as in figure $1 \mathrm{~B}$. compared with that on GlcNAc-BSA-coated plates. The addition of Fbg to the serum enhanced $\mathrm{C} 4$ deposition on the GlcNAc-BSA-coated plates in a dose-dependent manner (fig. 3b). The enhanced effect of Fbg was largely amplified by adding thrombin to the reaction mixture. Fbg/ Fbn appeared to be effective in the physiological range of serum concentration.

Bindings of $r F c n A, r M B L-A$ and $r M B L-C$ to S. aureus were Enhanced by Fbg/Fbn

We next evaluated the effects of Fbg/Fbn using S. aureus as a non-self target. As shown in figure $4 \mathrm{a}$, the binding of $\mathrm{rFcn} A$ to the bacteria was enhanced in the presence of Fbg. The binding was further enhanced by the addition of thrombin to the reaction mixture. Similar but slightly distinct effects of $\mathrm{Fbg} / \mathrm{Fbn}$ were observed on the bindings of rMBL-A and rMBL-C to S. aureus. Fbg showed no or 
Fig. 3. a C4 deposition activity of WT mouse serum on Fbg-coated microtiter plates (open circle). The various amounts of pooled mouse serum were incubated in Fbg-coated plates at $37^{\circ} \mathrm{C}$ for $10 \mathrm{~min}$. In a comparison, C4 deposition activity on GlcNAc-BSAcoated (open square) and that on non-coated microtiter plates (closed circle) were determined. b Effects of Fbg (open square) and Fbn (Fbg plus thrombin; closed square) on C4 deposition activity of mouse serum on GlcNAc-BSA-coated microtiter plates. The various amounts of Fbg were mixed with $0.75 \mu \mathrm{l}$ per well of serum in the presence (closed square) or absence (open square) of $20 \mathrm{mU}$ of thrombin. The shaded area represents the physiological range of Fbg in the human serum, which was calculated from the amounts of serum and Fbg used.

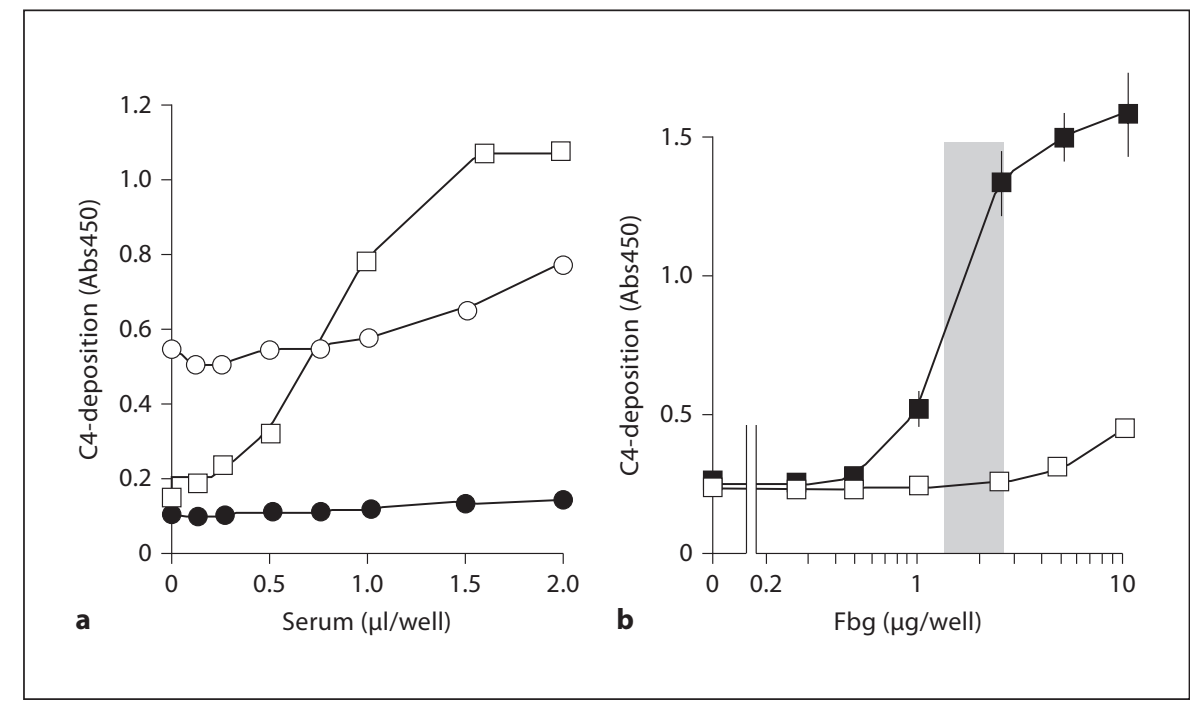

only a small effect on the bindings of rMBL-A and rMBL$\mathrm{C}$, while the presence of both Fbg and thrombin in the reaction mixture largely enhanced the bindings of rMBLs (Fig. 4b, c). This profile was similar to that in the bindings of rMBLs to Fbg-coated and Fbg-coated thrombin-treated plates, as described above (fig. $2 b$ ).

In an additional experiment to evaluate the effect of $\mathrm{Fbg} / \mathrm{Fbn}, \mathrm{S}$. aureus was pretreated with Fbg and Fbn (Fbg plus thrombin), and then subjected to binding with rFcnA. As compared with simultaneous mixing of $\mathrm{rFcnA}$ and $\mathrm{Fbg}$, the pretreatment with Fbg showed a reduced binding of $\mathrm{rFcnA}$ to the bacteria (fig. $4 \mathrm{~d}$, center panel), suggesting that a population of the bound Fbg is washed out before the incubation with $\mathrm{rFcnA}$. In contrast, pretreatment of $S$. aureus with Fbg and thrombin (fig. $4 \mathrm{~d}$, right panel) showed a very similar binding level to that observed by the simultaneous mixing. These results suggest that $\mathrm{rFcnA}$ binds to $\mathrm{Fbg} / \mathrm{Fbn}$ on the surface of bacteria in addition to the carbohydrate ligands.

\section{C3 Deposition on S. aureus was Enhanced in the \\ Presence of Fbg/Fbn}

C3 deposition on S. aureus was assessed using pooled mouse serum in the presence or absence of Fbg/Fbn. The addition of Fbg to the WT mouse serum enhanced C3 deposition on the bacteria (fig. 5a). The enhanced C3 deposition was amplified by the addition of thrombin. To evaluate the activities of FcnA-MASP and MBLs-MASP complexes, we assessed the $\mathrm{C} 3$ deposition on $S$. aureus using FcnA/MBL-depleted serum containing these complexes. As shown in figure 5b, C3 deposition activity of the mixture containing the FcnA/MBL-depleted serum and FcnA-MASPs complex was enhanced in the presence of Fbg, while the addition of thrombin had a limited effect on C3 deposition. In contrast, C3 deposition of the mixture containing the FcnA/MBL-depleted serum and the MBLs-MASPs complex was less enhanced by Fbg, but further addition of thrombin largely enhanced C3 deposition on the bacteria (fig. 5c).

\section{Discussion}

In the present study, we experimentally verified the interaction of $\mathrm{Fbg} / \mathrm{Fbn}$ with the recognition molecules of the lectin pathway, in which $\mathrm{rFcnA}$ and rMBLs directly bound to Fbg-coated plates, and Fbg/Fbn enhanced the bindings of $\mathrm{rFcnA}$ and $\mathrm{rMBLs}$ to $\mathrm{S}$. aureus and the subsequent activation of complements $\mathrm{C} 4$ and $\mathrm{C} 3$. We also found that $\mathrm{rFcnA}$ and rMBLs bind to the $\mathrm{A} \alpha$ - and $\mathrm{B} \beta$ chains of Fbg, and the $\alpha$ - and $\beta$-chains of Fbn, but not to the $\gamma$-chain. A difference was observed in the binding property between $\mathrm{rFcnA}$ and $\mathrm{rMBLs}$ : $\mathrm{rFcnA}$ binds to the 4 chains with similar affinities, while rMBLs preferentially bind to the $\alpha$-and $\beta$-chains. These binding profiles were observed throughout our present study, which included ELISA with Fbg/Fbn-coated microtiter plates, affinity Western blotting for Fbg/Fbn, and FACS for the binding to the bacteria. The subsequent complement activation showed a similar profile, which can be explained by the respective binding property of $\mathrm{rFcnA}$ or rMBLs (fig. 5b, c), or by the mixture of both (fig. 3b, 5a). 


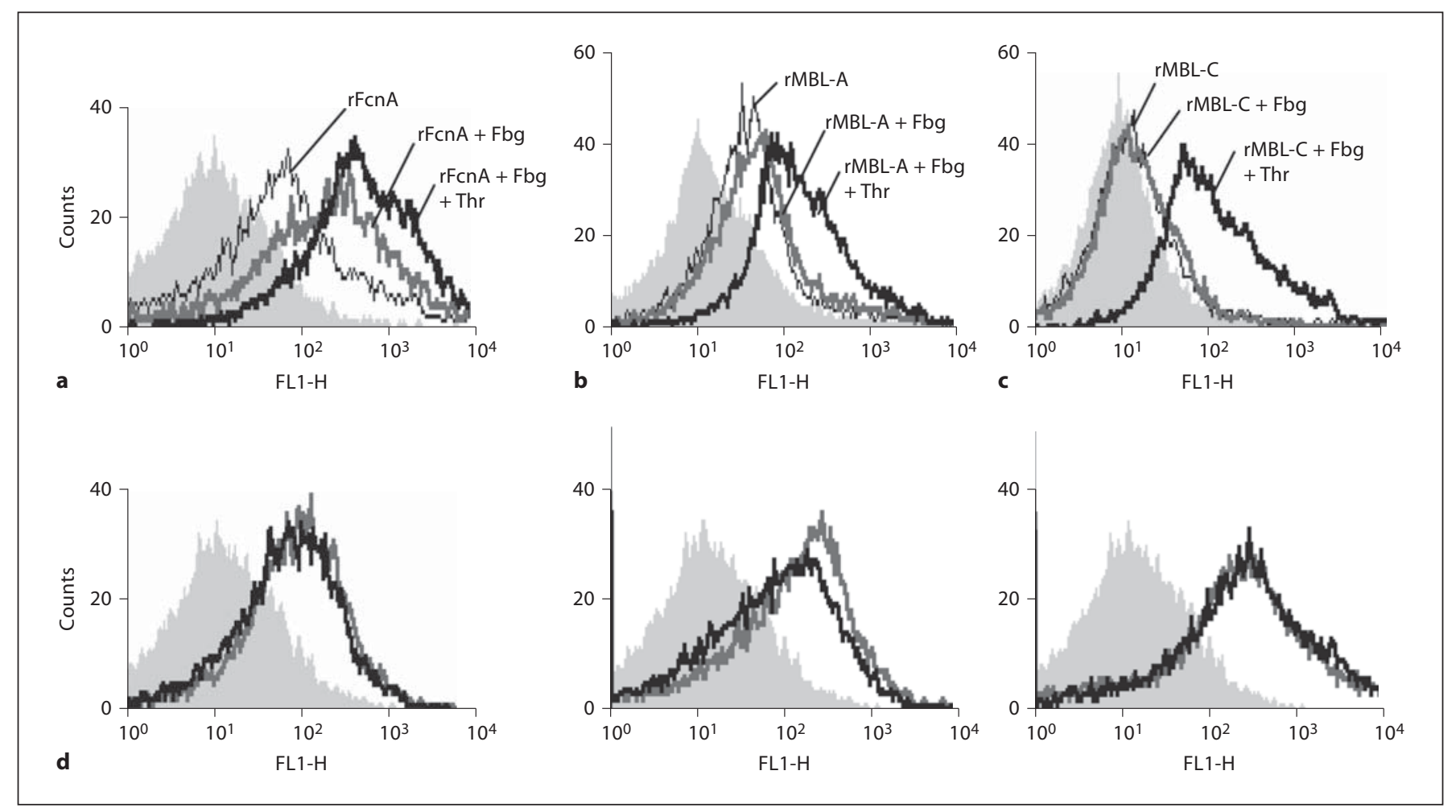

Fig. 4. Binding of rFcnA (a), rMBL-A (b) and rMBL-C (c) to $S$. aureus. The bindings were determined by FACS in the presence of Fbg (thick gray line) and Fbg plus thrombin (Thr) (thick black line). The binding without Fbg and thrombin is depicted with thin black lines, and the blank with the bacteria alone is by a shaded area in each figure. d Binding of $\mathrm{rFcnA}$ to $S$. aureus pretreated with Fbg. The bacteria were pretreated with $\mathrm{HBSS}^{++}$alone (left panel), Fbg (center panel) and Fbg plus thrombin (right panel), and then subjected to the binding assay with $\mathrm{rFcnA}$. The result obtained (thick black line) is compared with that by the simultaneous mixing as in a (thick gray line). The shaded area shows the results from blank with bacteria alone.



Fig. 5. a C3 deposition activity of WT mouse serum on S. aureus in the presence of Fbg (thick gray line) and Fbg plus thrombin (thick black line). WT mouse serum was incubated with $S$. aureus in the presence of Fbg (thick gray line) or Fbg plus thrombin (black thick line) as described in 'Materials and Methods'. In a control experiment, the $\mathrm{C} 3$ deposition activity was determined in the absence of Fbg and thrombin (thin black line). The shaded area shows the results from blank with the bacteria alone. b, c C3 deposition activity of the FcnA/MBL-depleted serum supplemented with the GlcNAc-eluate (b) or the mannose-eluate (c). The FcnA/MBL-depleted serum was mixed with Fbg, the GlcNAceluate or the mannose-eluate and $S$. aureus in the presence (thick black line) or absence (thick gray line) of thrombin (Thr). In a control experiment, C3 deposition activity was determined for the same mixture without Fbg (b, c, thin black line) and for the FcnA/MBL-depleted serum alone (b, $\mathbf{c}$, thin gray line). 
Fig.6. A schematic model showing the collaboration of the lectin pathway with blood coagulation. Each molecule of FCN or MBL binds to carbohydrates on the pathogen at the ligand-binding site(s) and also binds to $\mathrm{Fbg} / \mathrm{Fbn}$ at the remaining binding site(s). Thrombin and possibly MASP-2 activate factor XIII, and the activated factor XIII (XIIIa) crosslinks between Fbn and pathogen, in addition to between Fbn. These bindings (depicted with circles) form a network on the surface of pathogen, which stabilizes the recognition moleculetarget complex and therefore augments the lectin pathway by enhancing the activation of MASPs and the subsequent activation of complements such as C4, C2 and C3.

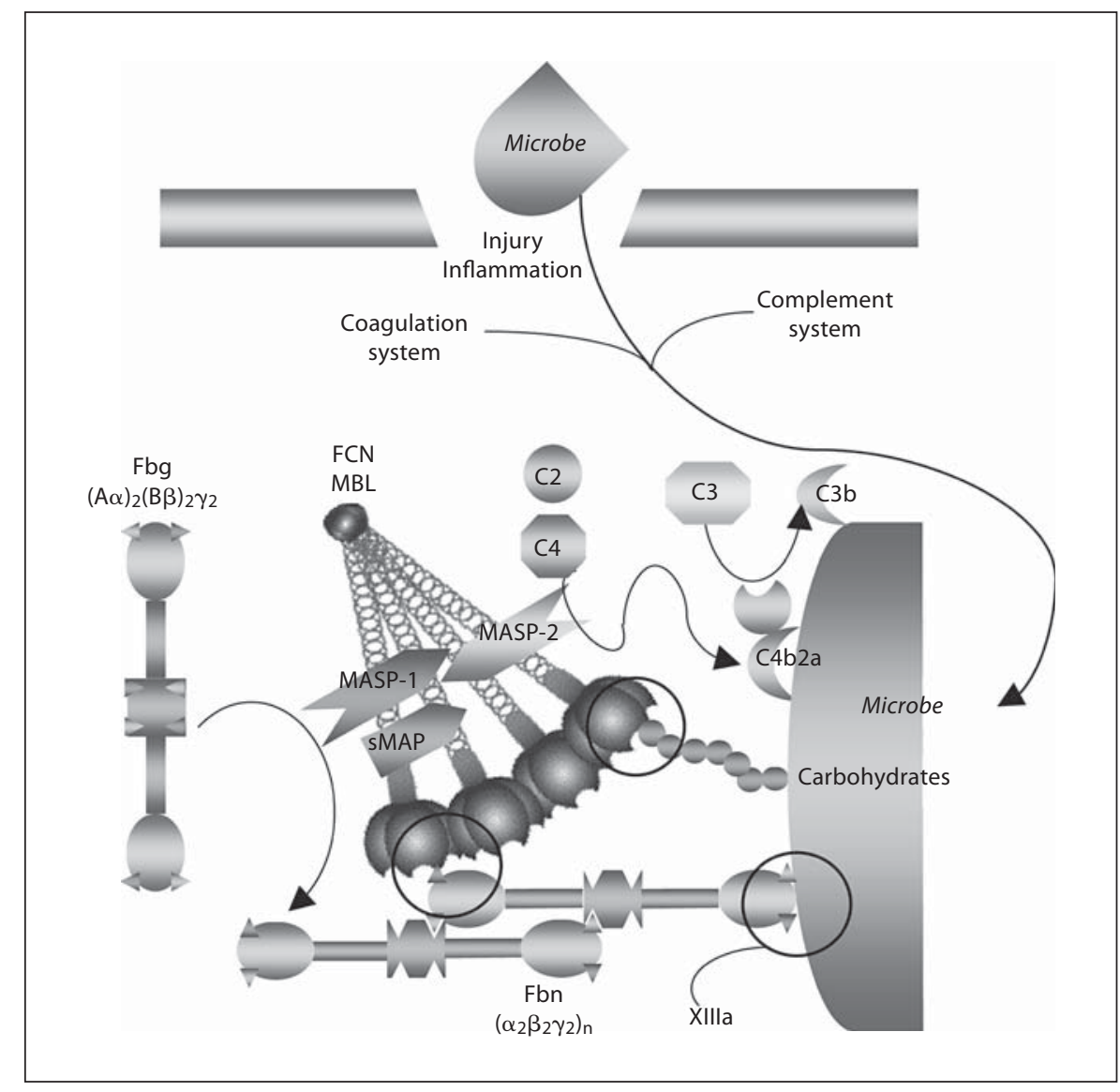

Our preliminary estimation of the concentrations of Fcn A and MBLs in mouse serum suggests that the amount of FonA is less than $10 \%$ of the total amount of MBL-A plus MBL-C (data not shown). Therefore, the complement activation of mouse serum would mainly reflect the sum total of the activities of MBLs rather than that of FcnA. The C4- and C3-deposition activities of WT mouse serum could be predominantly explored by MBLs, that explains the enhanced activity in the presence of Fbn.

It is well known that FCN recognizes the GlcNAc residue, but not the mannose residue, through its Fbg-like domain $[5,6,23]$, whereas MBLs recognize both mannose and GlcNAc residues through their carbohydrate recognition domain. Our inhibition experiments revealed that $\mathrm{rFcnA}$ recognizes the $A \alpha-, B \beta-, \alpha$ - and $\beta$ chains through the same or a similar site to the GlcNAcbinding site, because the binding of $\mathrm{rFcnA}$ to Fbg-coated microtiter plates and to $S$. aureus was inhibited by excess amounts of GlcNAc. Similarly, the bindings of rMBLs to $\mathrm{Fbg} / \mathrm{Fbn}$-coated microtiter plates were inhibited by GlcNAc and mannose, although the inhibition was in- complete. This suggests that rMBLs recognize the four chains through their carbohydrate recognition domain, which overlaps with the sugar-binding sites, with a preferential affinity for the $\alpha$ - and $\beta$-chains. Another important observation is that $\mathrm{rFcn} \mathrm{A}$ and $\mathrm{rMBL}$ recognize $\mathrm{Fbg} /$ Fbn not through the sugar moiety, because deglycosylation of Fbg did not affect their binding activity. Therefore, $\mathrm{rFcnA}$ and $\mathrm{rMBL}$ recognize some polypeptide regions of Fbg/Fbn through the Fbg-like and carbohydrate recognition domains, respectively, although the precise ligand sites on $\mathrm{Fbg} / \mathrm{Fbn}$ are unknown.

The difference in the binding mode between $\mathrm{rFcnA}$ and rMBLs suggests the distinct physiological significance committed to them. The strong binding of $\mathrm{rFcnA}$ to both Fbg and Fbn suggests that this interaction is constitutively ready for host defense against non-self and is dependent on Fbg, but independent of coagulation. In contrast, the preferential affinity of rMBLs to Fbn suggests that this interaction is induced at times when the coagulation is triggered. Thus, the interaction of the recognition molecules of the lectin pathway with $\mathrm{Fbg} / \mathrm{Fbn}$ 
represents 2 types of collaboration between the complement and the blood coagulation systems. Of particular note, the lectin pathway through MBLs appears to be tightly coupled with blood coagulation.

FCN and MBL are oligomers composed of homologous monomer subunits and therefore have multivalent ligand-binding sites $[9,24]$. Fbg is a dimer of 3 subunits, formulated as $(\mathrm{A} \alpha)_{2}(\mathrm{~B} \beta)_{2} \gamma_{2}$, and $\mathrm{Fbn}$ is a polymer consisting of $\left(\alpha_{2} \beta_{2} \gamma_{2}\right)_{\mathrm{n}}$. The interaction of 2 multivalent molecules, recognition molecules and Fbg/Fbn, would form a huge network overlapping targets, including pathogens. This network would serve as a powerful tool for host defense through preventing mobilization and infection of pathogens and ultimately leading to their phagocytosis and killing, especially in the cases such as injury, as illustrated in figure 6. It was reported that the commercially available Fbg preparation includes factor XIII as a contamination [25]. This might explain our present results, especially the result shown in figure $4 \mathrm{~d}$, where the mixing of $\mathrm{Fbg}$, thrombin and $S$. aureus possibly activated the contaminated factor XIII, in addition to the conversion of Fbg to Fbn. The activated factor XIII would cross-link covalently between Fbn and the bacteria [25] (fig. 6). In addition, Fbg-binding proteins, which have been identified in S. aureus [26, 27], probably contribute to anchor the network to the surface of the bacteria. Therefore, each molecule of FCN and MBL is allowed to bind to both carbohydrates and Fbn on the bacteria. The network formed on the surface of bacteria results in the enhanced bindings of FCN and MBL, enhanced activation of MASPs and complements, and severe opsonization of the bacteria with $\mathrm{C} 3 \mathrm{~b}$ and $\mathrm{C} 4 \mathrm{~b}$, which finally leads to the killing of bacteria.
Interestingly, it was reported that MASP-1, one of 3 kinds of MASP identified widely in vertebrates [28], showed thrombin-like activity $[29,30]$. The physiological meaning of this activity is unknown. It is also unknown whether this activity is specifically endowed to MASP-1, because MASP-1 appears to have a broad range of substrate specificity. If MASP-1 actually has this type of activity in vivo, it implies that the activated MASP-1 can initiate coagulation. It was also reported that MASP-2, which was identified in relatively higher vertebrates [28], was capable of forming Fbn by cleaving prothrombin and factor XIII [25]. These observations suggest another collaboration between the lectin pathway and coagulation system. Because MASP-1 and MASP-2 form complexes with both FCN and MBLs, the reported activities of MASP-1 and MASP-2 could be fully displayed in close contact with $\mathrm{Fbg} / \mathrm{Fbn}$. Thus, it is possible that the lectin pathway and the coagulation system collaborate by initiating each other in a bidirectional manner.

It has also been reported that the purified human Creactive protein can bind to Salmonella enterica and L$\mathrm{FCN}$, and that the interaction of $\mathrm{C}$-reactive protein with L-FCN stabilized the binding of CRP to the bacteria, which therefore increased complement activation [31, 32]. This observation is a close analogy to our present study, in that the recognition molecule-pathogen complex is stabilized by another plasma protein and, in turn, the stabilization boosts the lectin pathway. Thus, in conjunction with the previous reports, the present study clearly indicates that the lectin pathway crosstalks and collaborates with other defense systems such as blood coagulation and acute phase inflammation, to eliminate non-self with high efficiency.

\section{References}

$\checkmark 1$ Matsushita M, Fujita T: Activation of the classical complement pathway by mannosebinding protein in association with a novel C1s-like serine protease. J Exp Med 1992; 176:1497-1502.

$>2$ Matsushita M, Endo Y, Fujita T: Complement activation complex of ficolin and mannose-binding lectin-associated serine protease. J Immunol. 2000;164:2281-2284.

$>3$ Fujita T: Evolution of the lectin-complement pathway and its role in innate immunity. Nat Rev Immunol 2002;2:346-353.

4 Fujita T, Matsushita M, Endo Y: The lectincomplement pathway: its role in innate immunity and evolution. Immunol Rev 2004; 198:185-202.
5 Matsushita M, Endo Y, Taira S, Sato Y, Fujita T, Ichikawa N, Nakata M, Mizuochi T: A novel human serum lectin with collagenand fibrinogen-like domains that functions as an opsonin. J Biol Chem 1996;271:24482454.

6 Garlatti V, Belloy N, Martin L, Lacroix M, Matsushita M, Endo Y, Fujita T, FontecillaCamps JC, Arlaud GJ, Thielens NM, Gaboriaud C: Structural insights into the inanate immune recognition specificities of L- and H-ficolins. EMBO J 2007;26:623-633.

7 Endo Y, Sato Y, Matsushita M, Fujita T: Cloning and characterization of the human lectin P35 gene and its related gene. Genomics 1996;36:515-521.
$>8$ Lu J, Tay PN, Kon OL, Reid KBM: Human ficolin: cDNA cloning, demonstration of peripheral blood leucocytes as the major site of synthesis and assignment of the gene to chromosome 9. Biochem J 1996;313:473-478.

-9 Sugimoto R, Yae Y, Aikawa M, Kitajima S, Shibata Y, Sato H, Hirata J, Okochi K, Izuhara $\mathrm{K}$, Hamasaki N: Cloning and characterization of the Hakata antigen, a member of the ficolin/opsonin p35 lectin family. J Biol Chem 1998;273:20721-20727.

10 Fujimori Y, Harumiya S, Fukumoto Y, Miura Y, Yagasaki K, Tachikawa H, Fujimoto D: Molecular cloning and characterization of mouse ficolin-A. Biochem Biophys Res Commun 1998;244:796-800. 
11 Ohashi T, Erickson HP: Oligomeric structure and tissue distribution of ficolins from mouse, pig and human. Arch Biochem Biophys 1998;360:223-232.

12 Endo Y, Matsushita M, Fujita T: Role of ficolin innate immunity and its molecular basis. Immunobiology 2007;212:371-379.

13 Matsushita M, Kuraya M, Hamasaki N, Tsujimura M, Shirai H, Fujita T: Activation of the lectin complement pathway by $\mathrm{H}$-ficolin (Hakata antigen). J Immunol 2002;168: 3502-3506.

14 Liu Y, Endo Y, Nakata M, Wada I, Iwaki D, Inoue $\mathrm{K}$, Matsushita $\mathrm{M}$, Munakata $\mathrm{M}$, Fujita $\mathrm{T}$ : Human M-ficolin is a secretory protein that activates the lectin complement pathway. J. Immunol 2005;175:3150-3156.

$\checkmark 15$ Endo Y, Nakazawa N, Liu Y, Iwaki D, Takahashi M, Nakata M, Matsushita M, Fujita T: Carbohydrate-binding specificities of mouse ficolin A, splicing variant of ficolin A and ficolin B and their complex formation with MASP-2 and sMAP. Immunogenet 2005;57: 837-844.

16 Tanio M, Kondo S, Sugio S, Kohno T: Trivalent recognition unit of innate immunity system; crystal structure of trimeric human M-ficolin fibrinogen-like domain. J Biol Chem 2007;282:3889-3895.

17 Garlatti V, Martin L, Gout E, Reister J-B, Fujita T, Arlaud GJ, Thielens NM, Gaboriaud C: Structural basis for innate immune sensing by $\mathrm{M}$-ficolin and its control by a $\mathrm{pH}$-dependent conformational switch. J Biol Chem 2007;282:35814-35820.
18 Kairies N, Beisel H-G, Fuentes-Prior P, Tsuda R, Muta T, Iwanaga S, Bode W, Huber R, Kawabata S: The 2.0-A crystal structure of tachylectin $5 \mathrm{~A}$ provides evidence for the common origin of the innate immunity and the blood coagulation systems. Proc Natl Acad Sci USA 2001;98:13519-13524.

19 Endo Y, Liu Y, Kanno K, Takahashi M, Matsushita M, Fujita T: Identification of the mouse $\mathrm{H}$-ficolin gene as a pseudogene and orthology between mouse ficolins $\mathrm{A} / \mathrm{B}$ and human L-/M-ficolins. Genomics 2004;84: 737-744.

20 Kenjo A, Takahashi M, Matsushita M, Endo Y, Nakata M, Mizuochi T, Fujita T: Cloning and characterization of novel ficolins from the solitary ascidian, Halocynthia roretzi. Biol Chem 2001;276:19959-19965.

21 Okada N, Harada R, Fujita T, Okada H: A novel membrane glycoprotein capable of inhibiting membrane attack by homologous complement. Int Immunol 1989;1:205-208.

22 Iwaki D, Kanno K, Takahashi M, Endo Y, Lynch NJ, Schwaeble WJ, Matsushita M, Okabe M, Fujita T: Small mannose-binding lectin-associated protein plays a regulatory role in the lectin complement pathway. J Immunol 2006;177:8626-8632.

-23 Krarup A, Thiel S, Hansen A, Fujita T, Jensenius J C: L-ficolin is a pattern recognition molecule specific for acetyl groups. J Biol Chem 2003;279:47513-47519.

24 Ohashi T, Erickson HP: Two oligomeric forms of plasma ficolin have differential lectin activity. J Biol Chem 1997;272:1422014226.

-25 Krarup A, Wallis R, Presanis JS, Gál P, Sim RB: Simultanous activation of complement and coagulation by MBL-associated serine protease 2. PloS ONE 2007;2:e623.
26 McDevitt D, Francois P, Vaudaux P, Foster T: Molecular characterization of the clumping facter (fibrinogen receptor) of Staphylococcus aureus. Mol Microbiol 1994;11:237-248.

27 Wann E, Gurusiddappa S, Höök M: The fibronectin-binding MSRAMM FnbpA of Staphylococcus aureus is a bifunctional protein that also binds to fibrinogen. J Biol Chem 2000;275:13863-13871.

28 Endo Y, Nonaka M, Saiga H, Kakinuma Y, Matsushita A, Takahashi M, Matsushita M, Fujita T: Origin of mannose-binding lectinassociated serine protease (MASP)- 1 and MASP-3 involved in the lectin complement pathway traced back to the invertebrate, amphioxus. J Immunol 2003;170:4701-4707.

29 Hajela K, Kojima M, Ambrus G, Wong KH, Moffatt BE, Ferluga J, Hajela S, Gál P, Sim RB: The biological functions of MBL-associated serine proteases (MASPs). Immunobiology 2002;205:467-475.

30 Krarup A, Kojima M, Ambrus G, Nicky Wong K H, Moffatt BE, Ferluga J, Hajela S, Gál P, Sim RB: The action of MBL-associated serine protease 1 (MASP1) on factor XIII and fibrinogen. Biochim Biophys Acta 2008; 1784:1294-1300

31 Ng PML, Le Saux A, Lee CM, Tan NS, Lu J, Thiel S, Ho B, Ding JL: C-reactive protein collaborates with plasma lectins to boost immune response against bacteria. EMBO J 2007;26:3431-3440.

32 Zhang J, Koh J, Lu J, Thiel S, Leong BSH, Sethi S, He CYX, Ho B, Ding JL: Local inflammation induces complement crosstalk which amplifies the antimicrobial response. PLoS Pathog 2009;5:e10000282. 